\title{
Additions to the List of Polypores to India
}

\author{
Brij Bala, Avneet Pal Singh*, Gurpaul Singh Dhingra \\ Department of Botany, Punjabi University, India
}

Received October 31, 2019; Revised December 11, 2019; Accepted December 17, 2019

Copyright $\bigcirc 2020$ by authors, all rights reserved. Authors agree that this article remains permanently open access under the terms of the Creative Commons Attribution License 4.0 International License

\begin{abstract}
Five species of the pileate polypore fungi, Ganoderma ungulatum (Polyporales, Ganodermataceae), Gloeophyllum odoratum (Gloeophyllales, Gloeophyllaceae) Heterobasidion abietinum (Russulales, Bondarzewiaceae), Osmoporus mexicanus (Gloeophyllales, Gloeophyllaceae) and Oxyporus ravidus (Hymenochaetales, Schizoporaceae) are described and illustrated on the basis of basidiocarps collected during the rainy season of years 2015-2017 from different parts of Doda district (Union Territory of Jammu and Kashmir, India). Of these, the first four species are being described and illustrated for the first time from India, whereas Oxyporus ravidus is a new record for Union Territory of Jammu and Kashmir. Being the key wood decayers, these fungi grow in association with both broad-leaved as well as conifer tree species. Of the species described, the basidiocarps of Ganoderma ungulatum were collected from Quercus sp., whereas rest of the four species were growing in association with gymnospermous wood. Among the five species described presently, Heterobasidion abietinum is a host specific polypore that grows only in association with wood of Abies spp. These polypores are responsible for different types of rot and play a significant role in the recycling of different types of material. Of the described species, Ganoderma ungulatum Heterobasidion abietinum and Oxyporus ravidus are reported to cause white rot whereas Gloeophyllum odoratum and Osmoporus mexicanus are responsible for brown rot.
\end{abstract}

Keywords Basidiomycota, Poroid Fungi, White Rot, Brown Rot, North Western Himalaya

\section{Introduction}

Polypores (Agaricomycetes, Basidiomycota) are characteristic in having annual to perennial, resupinate to effused-reflexed to pileate, sessile to stipitate basidiocarps. These fungi have unilateral hymenium organized inside tubes which open through pores that can be circular, angular, daedaleoid, lamellate or irregular. In case of pileate members the pilear surface can be smooth, tuberculate, warted, scrupose, tomentose, velutinate, hirsute, hispid, etc. The colour of hymenial and abhymenial surfaces varies from whitish to some shades of yellow, orange, grey, violet, blue or red. These fungi play significant role in the forest ecosystem because of their ability to secrete lignin and cellulose degrading enzymes. Besides their role in recycling of carbon, some of these fungi have also been used in the traditional medicinal system [1].

Jammu division of Union Territory of Jammu and Kashmir, the area under investigation of the present studies, offers a wide range of variation in altitude and climate. The division has about $45.89 \%$ of its geographical area under forest cover. The vegetation can be broadly categorized into subtropical dry evergreen forests (dominated by Acacia catechu, Dalbergia sissoo, Eucalyptus sp., Dendrocalamus strictus etc.), sub-tropical pine forests (mainly Pinus roxburghii), Himalayan dry temperate forests (Abies pindrow, Acer sp. Aesculus indicus, Cedrus deodara, Juglans regia, etc.), Himalayan moist temperate forests (Abies pindrow, Cedrus deodara, Pinus wallichiana, Picea smithiana etc.), subalpine forests (Abies pindrow, Populus ciliata, Betula utilis, Rhododendron spp., Quercus spp. etc.) and alpine vegetation (Berberis spp., Geranium, Lonicera, etc.). The variation in altitude, vegetation and climate offer ideal set of conditions for the growth of polypore fungi. The previous workers reported 40 species of the polypore fungi $[2,3,4,5,6,7]$ from Jammu and Kashmir.

Keeping in view the variation in geography and climate; diversity of the tree species and few polypore species reported, the present studies were proposed and the localities of Jammu division were surveyed for the collection of polypore basidiocarps. Presently five polypore species i.e. Ganoderma ungulatum J.D. Zhao \& X.Q. Zhang, Gloeophyllum odoratum (Wulfen) Imazeki, Heterobasidion abietinum Niemelä \& Korhonen, Osmoporus mexicanum (Mont.) Y.C. Dai \& S.H. He, are 
described from different areas of Jammu. All of these, except Oxyporus ravidus (new for Jammu and Kashmir) are reported for the first time from India.

\section{Material and Methods}

The polypore basidiocarps were collected during the excursions carried out in various parts of district Doda of Jammu Division in the rainy months (July-September) of years 2014-2017. These basidiocarps were separated carefully from their substratum using a hammer and chisel. The macromorphological details i.e. nature of the basidiocarp, mode of attachment, hymenial and abhymenial surface, margins, etc. were recorded. A piece of the fertile portion of the basidiocarp was used for getting the spore print on a micro slide. After drying (in sun or on an electric drier), the collected basidiocarps were packed in ziplock airtight bags. The micro morphological characters were studied by making preparations in water, $3 \% / 5 \% /$ $10 \% \mathrm{KOH}, 1 \%$ phloxine, $1 \%$ Congo red and $1 \%$ cotton blue (in distilled water/lactophenol). The cyanophilous and amyloid reaction of different microscopic structures were studied in $1 \%$ cotton blue and Melzer's reagent (Iodine 0.5 $\mathrm{g}$, Potassium Iodide $1.5 \mathrm{~g}$, Chloral hydrate $20.0 \mathrm{~g}$ and distilled water $20.0 \mathrm{ml}$ ) respectively. The line diagrams of the microscopic structures were drawn with the help of a camera lucida mounted on a compound microscope at $100 \mathrm{X}, 400 \mathrm{X}$, and $1000 \mathrm{X}$ magnification. Finally the specimens were identified on the basis of comparison of the description with the literature and online repository [2, $3,4,5,6,7,8,9,10,11,12,13,14,15,16]$. The identified specimens were finally submitted to the Herbarium, Department of Botany, Punjabi University, Patiala (PUN) using standard packing protocol.

\section{Results}

Five polypore species spread over five genera, five families and five orders of Agaricomyectes (Basidiomycota) are described from Jammu division.

\subsection{Ganoderma ungulatum J.D. Zhao \& X.Q. Zhang, Acta mycol. sin.: 19, 1984}

Basidiocarp perennial, pileate, sessile, broadly attached, imbricate, ungulate; pieli up to $12 \times 10 \times 9.2 \mathrm{~cm}$ (length $\times$ breadth $\times$ thickness); abhymenial surface non-laccate, sulcate, white when fresh, becoming brownish on drying; hymenial surface poroid, reddish white to brownish white when fresh, not changing much on drying; pores round to angular, 5-6 per $\mathrm{mm}$; dissepiments up to $45 \mu \mathrm{m}$ thick; context homogenous, up to $7.5 \mathrm{~cm}$ thick, dark brown, with many crustaceous layers; tube layer up to $1.7 \mathrm{~cm}$ in depth; margins obtuse, concolorus on both hymenial and abhymenial side, sterile up to $3 \mathrm{~mm}$ on hymenial side.

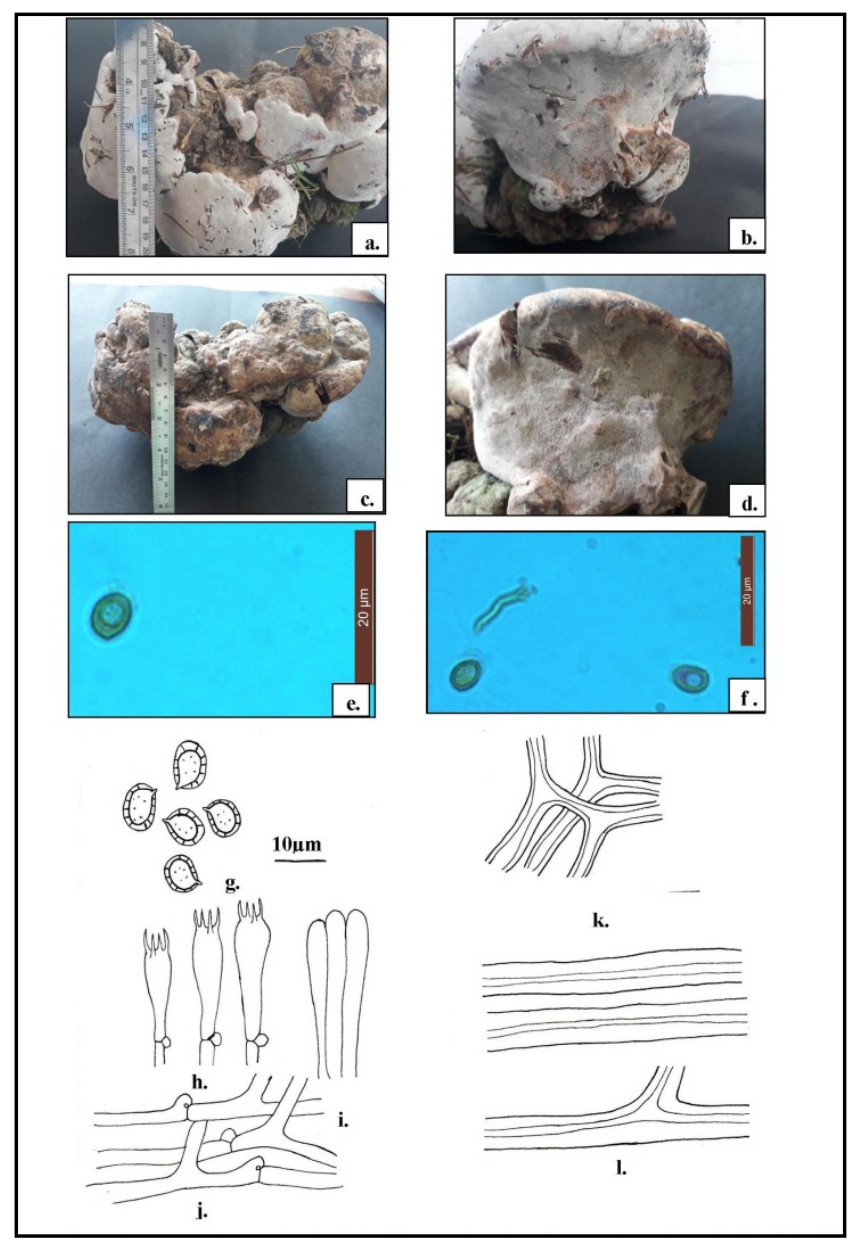

Figure 1 (a-l). Ganoderma ungulatum: (a-b) Fresh basidiocarp showing abhymenial and hymenial surface, (c-d) Dry basidiocarps showing abhymenial and hymenial surface, (e-f) photomicrographs showing basidiospores, (g) basidiospores, (h) basidia, (i) cuticular elements (j) generative hyphae, (k) binding hyphae, (l) skeleto-binding hyphae.

Pilear crust subanamixodermis, composed of hyaline, branched, agglutinated generative hyphae and solid, yellow, aseptate, branched or unbranched, skeletal hyphae.

Hyphal System trimitic. Generative hyphae thin-walled, clamped, branched, up to $4 \mu \mathrm{m}$ in width. Binding hyphae thick-walled, aseptate, irregularly branched, up to $4.5 \mu \mathrm{m}$ in width; skeletobinding hyphae thick-walled, occasionally branched, aseptate, up to $4 \mu \mathrm{m}$ in width. Cystidia absent. Basidia clavate, thin-walled, tetrasterigmate, basally clamped, $11.5-22 \times 4-5 \mu \mathrm{m}$; sterigmata upto $2 \mu \mathrm{m}$ in length. Basidiospores ovoid to ellipsoid, truncate, with thin, smooth exospores, thick, echinulate endospores, guttulate, acyanophilous, inamyloid, 6-7.5 × 3.5-5.8 $\mu \mathrm{m}$.

Collection examined: Jammu and Kashmir, Doda, Bhaderwah, Vasuki Naag Mandir Gattha, on Quercus branch, Brij Bala 9591(PUN), September 22, 2017.

Remarks: It is characterized by ungulate basidiocarps with non-laccate abhhymenial surface and thick, homogenous, dark brown context with many crustaceous layers. Earlier it is reported only from its type locality in 
China s $[8,13]$ on a stump of deciduous tree. This is the first report of $G$. ungulatum from India.

\subsection{Gloeophyllum odoratum (Wulfen) Imazeki, Bulletin of the Tokyo Science Museum 6: 75, 1943}

- Boletus odoratus Wulfen, Collectanea ad botanicam, chemiam, et historiam naturalem spectantia 2: 150, 1788.

Basidiocarp annual, pileate, sessile, broadly attached, ungulate, with anise odour; pieli up to $4.5 \times 2 \times 1.5 \mathrm{~cm}$; abhymenial surface tomentose, azonate, brown to dark brown when fresh, not changing much on drying; hymenial surface poroid, brown to light brown towards margins when fresh, not changing much on drying; pores angular to sinuous, 6-7 per $\mathrm{cm}$; dissepiments up to $67 \mu \mathrm{m}$ thick; context homogenous, brown, up to $1 \mathrm{~cm}$ thick; tube layers up to $0.5 \mathrm{~cm}$ in depth; margins obtuse, entire, concolorus both on hymenial and abhymenial surface, sterile up to $1 \mathrm{~mm}$ on hymenial side.

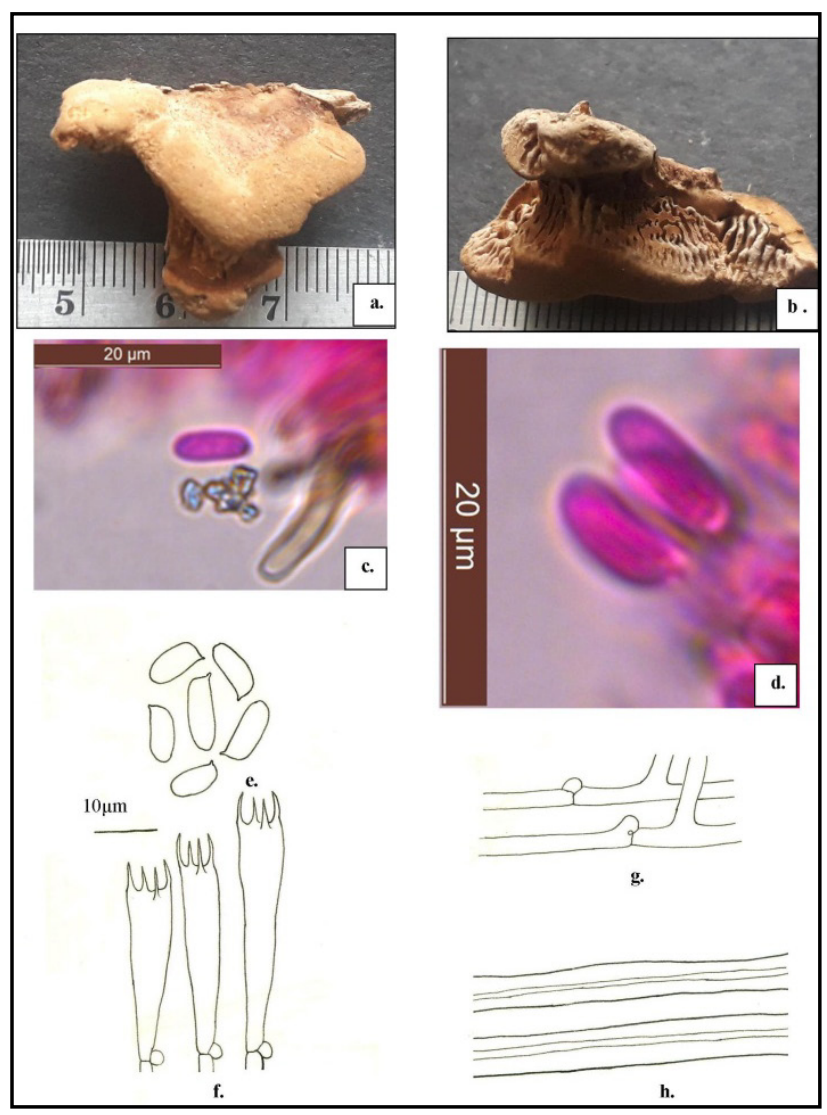

Figure 2(a-h). Gloeophyllum odoratum: (a-b) Basidiocarp showing abhymenial surface and hymenial surface, (c-d) photomicrographs showing basidiospores, (e) basidiospores, (f) basidia (g) generative hyphae, (h) skeletal hyphae.

Hyphal System dimitic. Generative hyphae thin- to thick-walled, clamped, branched, up to $4 \mu \mathrm{m}$ in width. Skeletal hyphae thick-walled, aseptate, unbranched, pale yellowish, up to $6 \mu \mathrm{m}$ in width. Basidia clavate, thin-walled, tetrasterigmate, basally clamped, $23-35 \times 8-9$ $\mu \mathrm{m}$; sterigmata upto $4 \mu \mathrm{m}$ in length. Basidiospores subcylindrical to cylindrical, thin-walled, acyanophilous, inamyloid, 7-12 × 2-3.5 $\mu \mathrm{m}$.

Collection examined: Jammu and Kashmir, Doda, Bhaderwah, Jai, on Picea stump, Brij Bala 9644 (PUN), September 26, 2015.

Remarks G. odoratum is peculiar in having smaller, ungulate pieli with charactrstic odour of anise seed. It is earlier known from North and Central Europe [13] and is being described for the first time from India.

\subsection{Heterobasidion abietinum Niemelä \& Korhonen. In: S. Woodw. et al., Heterobasidion annosum: Biology, Ecology, Impact and Control: 32, 1998}

Basidiocarp annual, pileate, imbricate, dimidiate, applanate; pilei up to $6 \times 4 \times 0.4 \mathrm{~cm}$; abhymenial surface faintly concentrically zonate, sulcate, white to violet brown when fresh, changing to pale yellow to greyish brown on drying; hymenial surface poroid, white to orange white when fresh, changing to pale yellow on drying; pores round to angular, 2-3 per mm; dissepiments up to $54 \mu \mathrm{m}$ thick; context homogenous, pale yellowish, up to $2 \mathrm{~mm}$ in thickness; tube layer up to $2 \mathrm{~mm}$ in depth; margins acute, entire, curved inside on drying, concolorous on both abhymenial and hymenial surface, sterile up to $1 \mathrm{~mm}$ on hymenial surface.

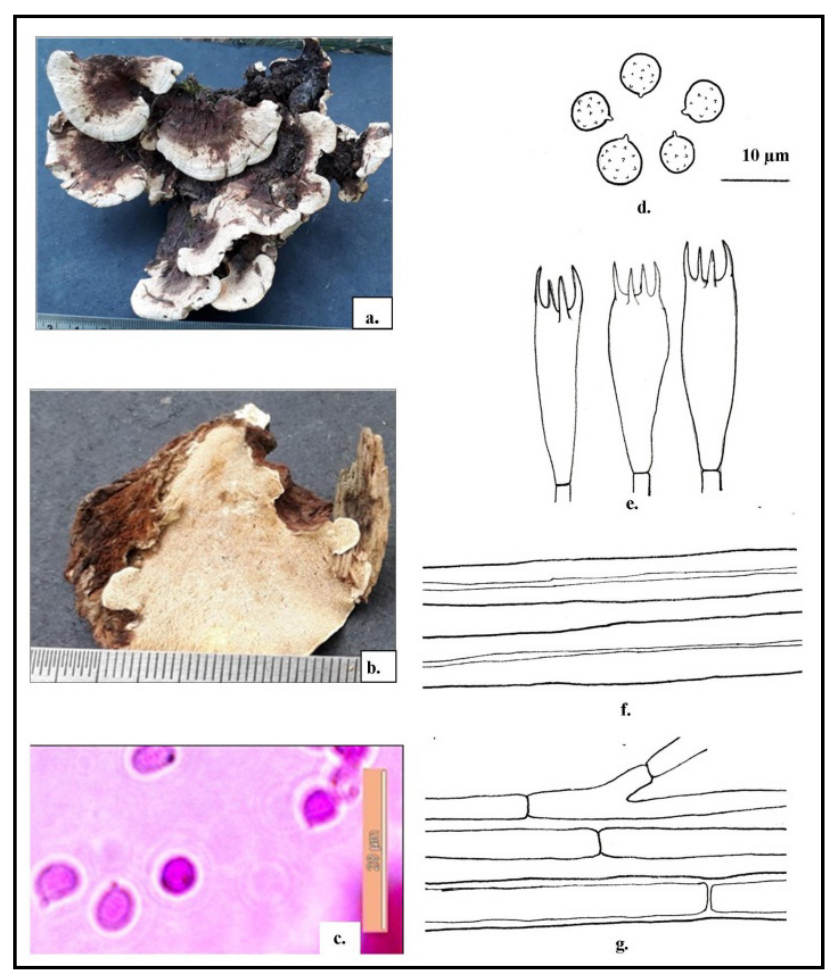

Figure 3 (a-g). Heterobasidion abeitinum: (a-b) Basidiocarp showings abhymenial and hymenial surface, (c) photomicrograph showing basidiospores, (d) basidiospores, (e) basidia, (f) skeletal hyphae, (g) generative hyphae.

Hyphal system dimitic. Generative hyphae thin-walled, simple-septate, branched, up to $5 \mu \mathrm{m}$ in width. Skeletal hyphae thick-walled, aseptate, unbranched, up to $6.5 \mu \mathrm{m}$ in width. Basidia clavate, thin-walled, tetrasterigmate, without basal clamp, $23-29 \times 7-8 \mu \mathrm{m}$; sterigmata up to 4 
$\mu \mathrm{m}$ in length. Basidiospores subglobse to globose, minutely echinulate, acyanophilous, inamyloid, 5-6 × 4-5 $\mu \mathrm{m}$.

Collection examined: Jammu and Kashmir, Doda, Shunushir on Abies pindrow stump, Brij Bala 9099 (PUN), September 21, 2016.

Remarks: $H$. abietinum is characteristic in having dimidiate, applanate, basidiocarps with comparatively larger pores and its unique association with Abies spp. It is distributed in Central Europe and Russia [9]. This is the first report of $H$. abietinum from India.

\subsection{Osmosporus mexicanus (Mont.)Y.C. Dai \& S.H. He, Mycological Progress 13 (3): 837, 2014. - Lenzites mexicana Mont., Annales des Sciences Naturelles Botanique 20: 360, 1843}

Basidiocarp annual, pileate, sessile, applanate, broadly attached; pilei up to $8.8 \times 5.4 \times 0.6 \mathrm{~cm}$; abhymenial surface sulcate, zonate, glabrous, greyish to light brown when fresh, not changing much on drying; hymenial surface greyish orange to brown when fresh, not changing much on drying; pores daedeloid to somewhat lamellate, 1-2 per mm; dissepiments up to $80 \mu \mathrm{m}$ thick; context homogenous, dark brown, up to $3 \mathrm{~mm}$

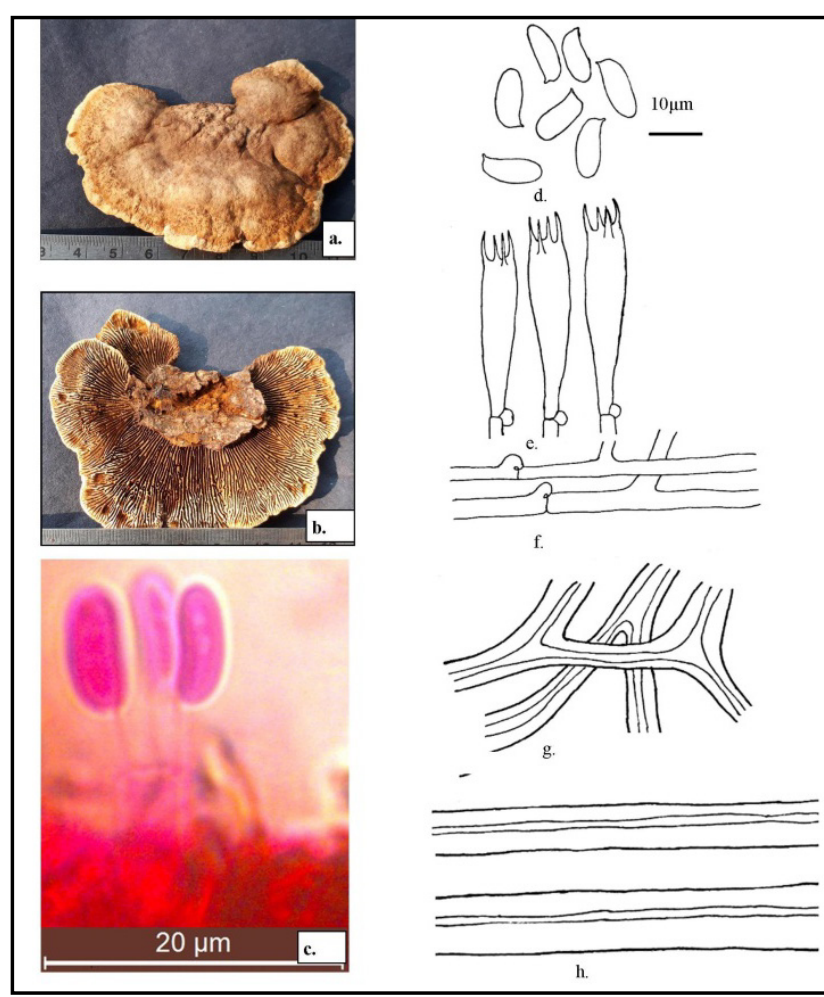

Figure 4 (a-h). Osmoporus mexicanus: (a-b) Basidiocarp showing abhymenial and hymenial surface, (c) photomicrograph showing basidiospores attached with basidia, (d) basidiospores, (e) basidia, (f) generative hyphae, (g) binding hyphae,(h) skeletal hyphae.

in thickness; tube layer up to $3 \mathrm{~mm}$ deep; margins acute, wavy, greyish red to greyish brown on abhymenial surface, pale orange to greyish orange on hymenial surface, sterile up to $1 \mathrm{~mm}$ on hymenial side.

Hyphal System trimitic. Generative hyphae thin- to thick-walled, clamped, branched, up to $5 \mu \mathrm{m}$ in width. Bindng hyphae thick-walled, branched up to $4.7 \mu \mathrm{m}$ in width. Skeletal hyphae thick-walled, aseptate, unbranched, up to $6 \mu \mathrm{m}$ in width. Basidia clavate, basally clamped, thin-walled, tetrasterigmate, up to $20.5-33 \times 5.5-7.5 \mu \mathrm{m}$; sterigmata up to $5 \mu \mathrm{m}$ in length. Basidiospores cylindrical, slightly curved, smooth, thin-walled, acyanophilous, inamyloid, 9.8-11 × 3-4 $\mu \mathrm{m}$.

Collection examined: Jammu and Kashmir, Doda, Bhaderwah, on Cedrus deodara stump, Brij Bala 9643 (PUN), September 25, 2016.

Remarks: It is distinct in having pileate basidicarps with glabrous abhymenial surface, daedeloid to lamellate pores and larger cylindrical basidispores. As per Mycobank [13] it is distributed in different parts of USA, Mexico and West Indies. Presently it is being described as a new record for India.

\subsection{Oxyporus ravidus (Fr.) Bondartsev \& Singer, Annales Mycologici 39 (1): 63, 1941. - Polyporus ravidus Fr., Epicrisis Systematis Mycologici: 475, 1838}

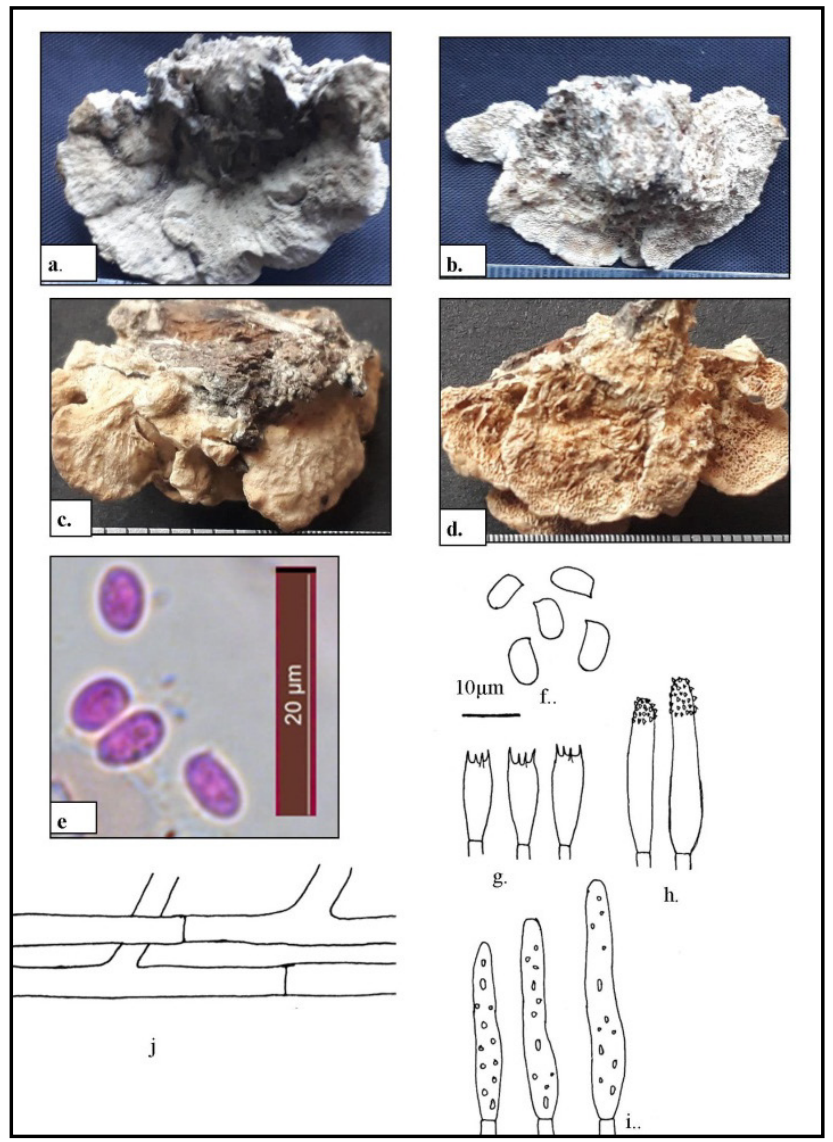

Figures 5(a-i). Oxyporus ravidus (a-b) Fresh basidiocarp showing abhymenial and hymenial surface (c-d) dry basidiocarp showing abhymenial and hymenial surface, (e) photomicrograph showing basidiospores, (f) basidiospores, (g) basidia, (h) cystidia, (i) gloeocystidia (j) generative hyphae. 
Basidiocarp annual, pileate, sessile, imbricate, dimidiate, applanate; pilei up to $2 \times 0.8 \times 0.4 \mathrm{~cm}$; abhymenial surface azonate, sulcate, orange white when fresh, not changing much on drying; hymenial surface poroid, orange grey when fresh, changing to pale yellow on drying; pores round to angular, 2-3 per mm; dissepiments up to $54 \mu \mathrm{m}$ thick; context homogenous, pale yellowish, up to $2 \mathrm{~mm}$ in thickness; tube layer up to $2 \mathrm{~mm}$ thick; margins acute, entire, concolorous on both abhymenial and hymenial surface, sterile up to $1 \mathrm{~mm}$ on hymenial side. Hyphal system monomitic. Generative hyphae thin-walled, simple-septate, branched, up to $5 \mu \mathrm{m}$ in width. Basidia clavate, thin-walled, tetrasterigmate, without basal clamp, 11.6-14.5 $\times 5.2-6.3 \mu \mathrm{m}$; sterigmata up to $2.5 \mu \mathrm{m}$ in length. Cystidia subcalavate thin-walled, apically encrusted, 25-32 $\times 4-5 \mu \mathrm{m}$. Gloeocystidia cylindrical to fusiform, thin-walled, with oily contents, arising in subhymenium, often projecting out of the hymenium, 33$45 \times 6-10 \mu \mathrm{m}$. Basidiospores ellipsoid, thin-walled, smooth, acyanophilous, inamyloid, 6-7 × 3-4 $\mu \mathrm{m}$.

Collection examined: Jammu and Kashmir, Doda, Shunushir on Cedrus deodara stump, Brij Bala 9642(PUN), September 21, 2016.

Remarks: It is characteristic in having large,r round to angular pores, monomitic hyphal system, two types of cystidia and larger, ellipsoid basidiospores. This is first report for the Union Territory of Jammu and Kashmir. It is commonly encountered polypore reported in association with both conifers broad leaved tree species in the temperate Himalayan region [10].

\section{Discussion}

The diverse climatic conditions of Union Territory of Jammu and Kashmir have attracted mycologists from different parts of India. As a result of their exploration of the union territory previous workers have reported 40 polypore species which are presently grouped under 23 genera, 8 families and 4 orders of Agaricomycetes (Basidiomycota) $[2,3,4,5,6]$. The present studies have added 4 new records for India and one new report for Jammu and Kashmir, thus raising the total number of polypore species from Jammu and Kashmir from 40 to 45 (Table 1). It is worth mentioning here that the genus Osmoporus is being described for the first time from Jammu and Kashmir based on O. mexixcanus.

Table 1. List of polypore species reported from Jammu and Kashmir

\begin{tabular}{|c|c|c|c|c|c|}
\hline S. no. & Name of the specie & Host & Systematic position & Locality \& Altitude (m) & Reference \\
\hline 1. & $\begin{array}{l}\text { Antrodia lenis } \\
\text { (as Poria lenis) }\end{array}$ & Log of Abies & $\begin{array}{c}\text { Polyporales, } \\
\text { Fomitopsidaceae }\end{array}$ & Gulmarg, $3747 \mathrm{~m}$ & {$[2],[3]$} \\
\hline 2. & A. serailis & Gymnospermic log & $\begin{array}{c}\text { Polyporales, } \\
\text { Fomitopsidaceae }\end{array}$ & Pahalgam $2740 \mathrm{~m}$ & {$[2],[3]$} \\
\hline 3. & Bjerkandera adusta & $\begin{array}{l}\text { On stump under mixed } \\
\text { forest }\end{array}$ & $\begin{array}{l}\text { Polyporales, } \\
\text { Meruliaceae }\end{array}$ & Batote. $1555 \mathrm{~m}$ & [3] \\
\hline 4. & Coltricia cinnamomea & Log of Cedrus deodara & $\begin{array}{c}\text { Hymenocahetale, } \\
\text { Hymenochaetaceae }\end{array}$ & Patnitop 2024 m & {$[3]$} \\
\hline 5. & C. perrenis & Log of Cedrus deodara & $\begin{array}{c}\text { Hymenocahetale, } \\
\text { Hymenochaetaceae }\end{array}$ & Sonmarg, 2800 & [3] \\
\hline 6. & Dadalea quercina & On stump of Quercus & $\begin{array}{c}\text { Polyporales, } \\
\text { Fomitopsidaceae }\end{array}$ & Patnitop 2024 m & {$[3],[6]$} \\
\hline 7. & Fomes fomentarius & On coniferous $\log$ & $\begin{array}{l}\text { Polyporales, } \\
\text { Polyporaceae }\end{array}$ & Bhaderwah, 1613 & [3] \\
\hline 8. & Fomitopsis rosea & On coniferous $\log$ & $\begin{array}{l}\text { Polyporales, } \\
\text { Polyporaceae }\end{array}$ & Bhaderwah, 1613 & [3] \\
\hline 9. & F. rufolaccata & On log of Abies pindrow. & $\begin{array}{l}\text { Polyporales, } \\
\text { Polyporaceae }\end{array}$ & Gulmarg, 3747 & [3] \\
\hline 10. & $\begin{array}{l}\text { Ganoderma } \\
\text { applanatum }\end{array}$ & On angiospermous wood. & $\begin{array}{c}\text { Polyporales, } \\
\text { Ganodermataceae }\end{array}$ & Pahalgam, 2740 & [3] \\
\hline 11. & G. lucidum & Base of Pinus excelsa & $\begin{array}{c}\text { Polyporales, } \\
\text { Ganodermataceae }\end{array}$ & Batote, 1555 & [3] \\
\hline 12. & G. resinaceum & Base of Platanus orientalis & $\begin{array}{c}\text { Polyporales, } \\
\text { Ganodermataceae }\end{array}$ & Srinagar, 1585 & {$[3],[5]$} \\
\hline 13. & G. ungulatum & Base of Quercus, & $\begin{array}{c}\text { Polyporales, } \\
\text { Ganodermataceae }\end{array}$ & Bhaderwah, 1613 & $\begin{array}{l}\text { New to } \\
\text { India }\end{array}$ \\
\hline 14. & G. tornatum & Unknown stump & $\begin{array}{c}\text { Polyporales, } \\
\text { Ganodermataceae }\end{array}$ & Pahalgam,27 40 & [3] \\
\hline 15. & $\begin{array}{l}\text { Gloeophyllum } \\
\text { sepiarium }\end{array}$ & Log of Cedrus deodara & $\begin{array}{l}\text { Gloeophyllales, } \\
\text { Gloeophyllaceae }\end{array}$ & Pahalgam,27 40 & [3] \\
\hline 16. & G. subferruginum & Log of Cedrus deodara & $\begin{array}{l}\text { Gloeophyllales, } \\
\text { Gloeophyllaceae }\end{array}$ & Gulmarg,3 747 & [3] \\
\hline 17. & G.odoratum & Log of Cedrus deodara & $\begin{array}{c}\text { Gloeophyllales, } \\
\text { Gloeophyllaceae }\end{array}$ & Jai, Bhaderwah, 2400. & $\begin{array}{l}\text { New to } \\
\text { India }\end{array}$ \\
\hline
\end{tabular}




\begin{tabular}{|c|c|c|c|c|c|}
\hline 18. & $\begin{array}{c}\text { Heterobasidion } \\
\text { abetinum }\end{array}$ & On log of Abies & $\begin{array}{c}\text { Russulales, } \\
\text { Bondarzewiaceae }\end{array}$ & $\begin{array}{c}\text { Shunushir, Bhaderwah, } \\
1613 .\end{array}$ & $\begin{array}{l}\text { New to } \\
\text { India }\end{array}$ \\
\hline 19. & $\begin{array}{l}\text { Heterobasidion } \\
\text { insularis }\end{array}$ & On stump of Pinus excelsa & $\begin{array}{c}\text { Russulales, } \\
\text { Bondarzewiaceae }\end{array}$ & Batote, 1555 & {$[3]$} \\
\hline 20. & Incrustoporia nivea & Dead log of Quercus & $\begin{array}{l}\text { Polyporales, } \\
\text { Polyporaceae }\end{array}$ & Bhaderwah, 1613. & {$[3]$} \\
\hline 21. & Inonotus dryadeus & Stump of Abies & $\begin{array}{c}\text { Hymenocahetale, } \\
\text { Hymenochaetaceae }\end{array}$ & Bhaderwah, 1613 & {$[3]$} \\
\hline 22. & Irpex lacteus & On log of Cedrus deodara & $\begin{array}{c}\text { Polyporales, } \\
\text { Phanerochaetaceae }\end{array}$ & Seoj Bhaderwah, 2400. & {$[3]$} \\
\hline 23. & I. zonatus & On log of Cedrus deodara & $\begin{array}{c}\text { Polyporales, } \\
\text { Phanerochaetaceae }\end{array}$ & Patnitop, 2024 & {$[3]$} \\
\hline 24. & Junghuhnia collabens & Unknown Stump & $\begin{array}{c}\text { Polyporales, } \\
\text { Phanerochaetaceae }\end{array}$ & Batote, 1555. & {$[3]$} \\
\hline 25. & Lenzites betulina & On stump of $Q$. incana & $\begin{array}{l}\text { Polyporales, } \\
\text { Polyporaceae }\end{array}$ & Batote, 1555 & {$[3]$} \\
\hline 26. & $\begin{array}{c}\text { Mycoleptodonoides } \\
\text { aitchisonii }\end{array}$ & $\begin{array}{c}\text { On stump of Cedrus } \\
\text { deodara }\end{array}$ & $\begin{array}{c}\text { Polyporales, } \\
\text { Phanerochaetaceae }\end{array}$ & Gulmarg, 3747 & {$[5]$} \\
\hline 27. & Osmoporus mexicanus & On $\log$ of C.deodara & $\begin{array}{c}\text { Polyporales, } \\
\text { Phanerochaetaceae }\end{array}$ & Bhaderwah, 1613 & $\begin{array}{l}\text { New to } \\
\text { India }\end{array}$ \\
\hline 28. & Onnia circinata & $\begin{array}{c}\text { Needles and Bark of Pinus } \\
\text { excelsa }\end{array}$ & $\begin{array}{l}\text { Hymenocahetale, } \\
\text { Hymenochaetaceae }\end{array}$ & Batote, 1555 & {$[3]$} \\
\hline 29. & $\begin{array}{l}\text { Oxyporus corticola } \\
\text { (as Poria corticola) }\end{array}$ & On stump of Abies. & $\begin{array}{l}\text { Hymenochaetales, } \\
\text { Schizoporaceae }\end{array}$ & Gulmarg, 3747 & {$[2],[3]$} \\
\hline 30. & Oxyporus ravidus & On log of Cedrus deodara & $\begin{array}{c}\text { Hymenochaetales, } \\
\text { Schizoporaceae }\end{array}$ & $\begin{array}{c}\text { Shunushir, Bhaderwah, } \\
2400\end{array}$ & $\begin{array}{l}\text { New to } \\
\text { India }\end{array}$ \\
\hline 31. & Phaeolus schwentizii & On base of C.deodara & $\begin{array}{c}\text { Hymenocahetale, } \\
\text { Hymenochaetaceae }\end{array}$ & Patnitop, 2024 & {$[3]$} \\
\hline 32. & Phellinus caryophylli & Stump under Pinus excelsa & $\begin{array}{c}\text { Hymenocahetale, } \\
\text { Hymenochaetaceae }\end{array}$ & Batote, 1555 & {$[3]$} \\
\hline 33. & Phellinus contigus & $\begin{array}{c}\text { Stump under mixed } \\
\text { forests. }\end{array}$ & $\begin{array}{c}\text { Hymenocahetale, } \\
\text { Hymenochaetaceae }\end{array}$ & Bhaderwah, 1613 & {$[2],[3]$} \\
\hline 34. & Phellinus linteus & $\begin{array}{l}\text { Stump and Base of Pinus } \\
\text { excelsa }\end{array}$ & $\begin{array}{l}\text { Hymenocahetale, } \\
\text { Hymenochaetaceae }\end{array}$ & Bhaderwah, 1613 & {$[3]$} \\
\hline 35. & Phellinus robustus & Stump of Abies & $\begin{array}{c}\text { Hymenocahetale, } \\
\text { Hymenochaetaceae }\end{array}$ & Gulmarg, 3747 & {$[3]$} \\
\hline 36. & Phellinus sanfordii & On angiospermic tree & $\begin{array}{c}\text { Hymenocahetale, } \\
\text { Hymenochaetaceae }\end{array}$ & Bhaderwah, 1613 & {$[3]$} \\
\hline 37. & Phellinus scropsus & Stump of Cedrus deodara & $\begin{array}{c}\text { Hymenocahetale, } \\
\text { Hymenochaetaceae }\end{array}$ & Batote, 1555 & {$[3]$} \\
\hline 38. & Ph. torulosus & On stump of Pinus excelsa & $\begin{array}{c}\text { Hymenocahetale, } \\
\text { Hymenochaetaceae }\end{array}$ & Batote, 1555 & \\
\hline 39. & Porodaedalea pini & Stump under Pinus excelsa & $\begin{array}{c}\text { Hymenocahetale, } \\
\text { Hymenochaetaceae }\end{array}$ & Batote, 1555 & {$[3]$} \\
\hline 40. & $\begin{array}{l}\text { Trametes hirsutus (as } \\
\text { Coriolus hirsutus) }\end{array}$ & $\begin{array}{c}\text { On stump under Cedrus } \\
\text { deodara }\end{array}$ & $\begin{array}{l}\text { Polyporales, } \\
\text { Polyporaceae } \\
\end{array}$ & Batote, 1555 & {$[3]$} \\
\hline 41. & $\begin{array}{l}\text { Trametes verscicolor } \\
\text { (as Coriolus versicolor) }\end{array}$ & On stump of Quercus & $\begin{array}{l}\text { Polyporales, } \\
\text { Polyporaceae }\end{array}$ & Batote, 1555 & {$[3]$} \\
\hline 42. & $\begin{array}{l}\text { Trametes zonatus (as } \\
\text { Coriolus zonatus) }\end{array}$ & On twig of Abies & $\begin{array}{l}\text { Polyporales, } \\
\text { Polyporaceae }\end{array}$ & Batote, 1555 & {$[3]$} \\
\hline 43. & $\begin{array}{l}\text { Trichaptum abietnum } \\
\text { (as Trichaptum } \\
\text { abietinus) } \\
\end{array}$ & On trunk of Berberis & $\begin{array}{l}\text { Polyporales, } \\
\text { Polyporaceae }\end{array}$ & Batote, 1555 & {$[3]$} \\
\hline 44. & $\begin{array}{c}\text { T. venusta } \\
\text { (as Trichaptum } \\
\text { venustum) }\end{array}$ & Stump of Quercus & $\begin{array}{l}\text { Polyporales, } \\
\text { Polyporaceae }\end{array}$ & Batote, 1555 & {$[3]$} \\
\hline 45. & $\begin{array}{l}\text { Wrightoporia lenta } \\
\quad \text { (Poria lenta })\end{array}$ & On log of Cedrus deodara & $\begin{array}{l}\text { Polyporales, } \\
\text { Polyporaceae }\end{array}$ & Bhaderwah, 1613 & {$[3],[4]$} \\
\hline
\end{tabular}




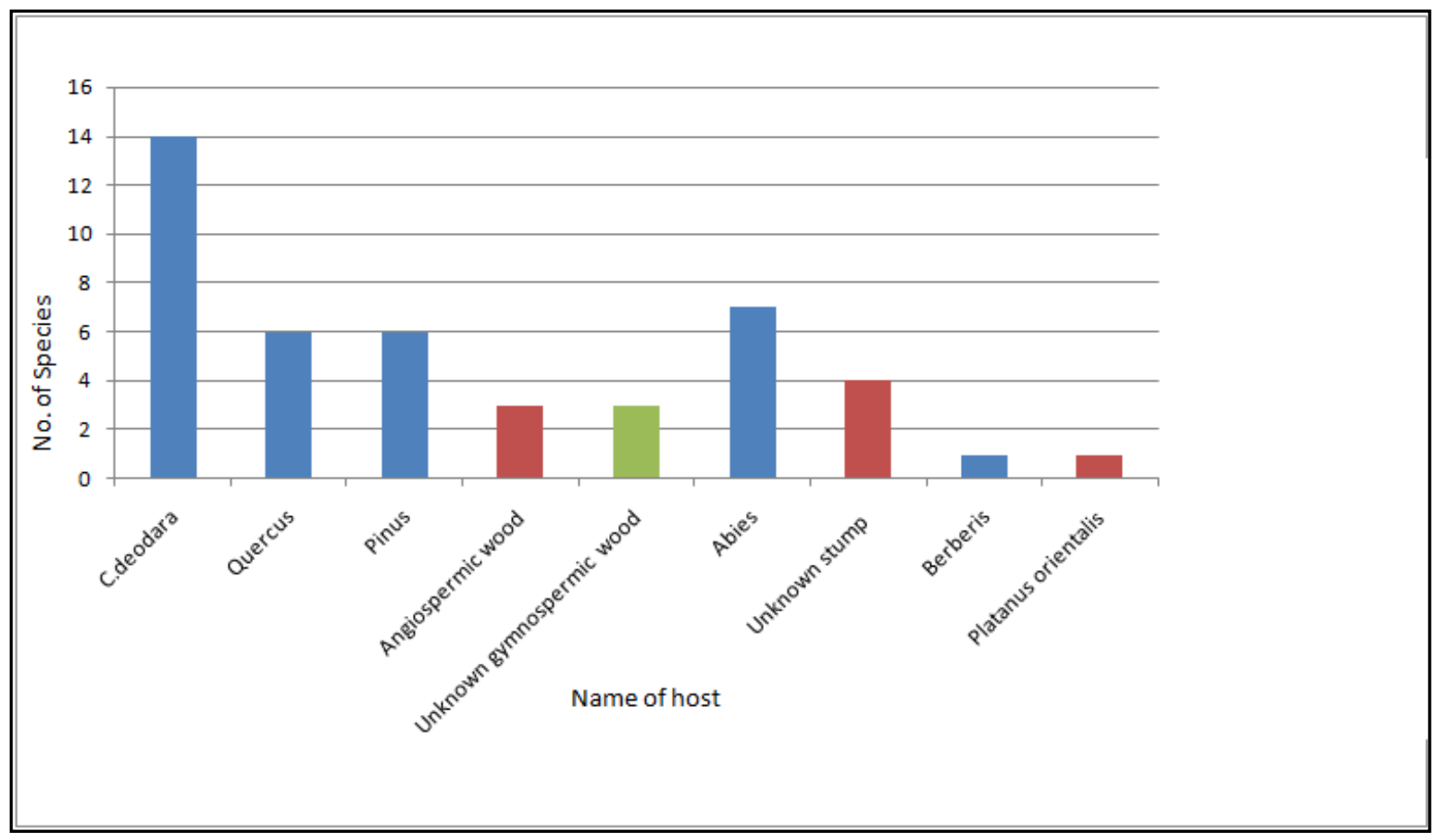

Figure 6. Host distribution of 45 species

Polypore fungi depend mainly on the wooden substrate for their nourishment. These fungi are unique in the ability to decay lignin, cellulose, hemicelluloses, etc. Hence they play a very significant role in recycling of materials. These fungi grow in association with both gymnospermous and angiospermous wood. Of the 45 species described from the study area, 30 species have been reported in association with gymnospermous trees/wood [Cedrus deodara (14), Abies (7), Pinus excelsa (6) and unidentified gymnospermous wood (3)], 11 species have been reported with angiospermous trees/wood [Quercus sp. (6), Berberis aristata (1), Platanus orientalis (1) and unidentified angiospermous wood (3)] and the remaining 4 species have been reported with unknown stumps (Figure 6).

As far as the geographical distribution is concerned, these fungi are dominant in the subtropical $(100-1800 \mathrm{~m})$ and temperate (1800-3600) regions. Among 45 species reported from the Jammu and Kashmir, 26 have been collected from the sub-tropical region, 13 from the temperate region and remaining 6 from the sub alpine region.

\section{Conclusions}

In the present scenario of changing climatic conditions it is essential to document the diversity of polypores of comparatively lesser explored Jammu and Kashmir. The knowledge of these fungi can lay a solid foundation for the exploitation of these fungi because of their medicinal importance. The present studies are one step forward in this direction. Further exploration will enrich our knowledge of these fungi form this diverse area.

\section{Acknowledgements}

The authors are grateful to the Head, Department of Botany, Punjabi University Patiala, for providing necessary laboratory facilities and University Grants Commission, New Delhi for financial assistance under UGC DRS DSA-I programme.

\section{REFERENCES}

[1] Y. Sone, R. Okuda, N. Wada, E. Kishida, and A. Misaki. Structure and antitumor activities of the polysaccharides isolated from fruiting body and the growing culture of mycelium of Ganoderma lucidum. Agricultural and Biological Chemistry, 49, 2641-2653, 2014.

[2] S.S. Rattan. The resupinate Aphyllophorales of North Western Himalayas. Bibliotheca Mycologica 60, 1-427, 1977.

[3] R.S. Dhanda. Studies on Polyporaceae of North Western Himalayas. Ph. D. Thesis, Panjab University, Chandigarh. $500 \mathrm{pp}$.

[4] K.S. Thind, R.S. Dhanda. The Polyporaceae of India-IX. Eight species of Poria new to India. Kavaka 7, 51-58, 1979.

[5] K.S. Thind, R.S. Dhanda. The Polyporaceae of India-X. Indian Phytopathology 8, 59-67, 1980a.

[6] K.S. Thind, R.S. Dhanda. The Polyporaceae of India-XIII. Indian Phytopathology 33(3), 80-387, 1980 b. 
[7] J.R. Sharma. Aphyllophorales of Himalaya (Auriscalpiaceae-Tremellodendropsis). Botanical Survey of India, Ministry of Environment \& Forests, Govt. of India, Calcutta, 1-590, 2012.

[8] J.D. Zhao. The Ganodermataceae in China. Bibliotheca Mycologica 132, 1-176, 1989.

[9] L. Ryvarden, I. Melo. Poroid fungi of Europe. Synopsis Fungorum, 31, 1-455, 2014.

[10] B. K. Bakshi. Indian Polyporaceae (on trees and timber). Indian Council of Agricultural Research, New Delhi, 1-246, 1971.

[11] A. Roy, A.B. De. Polyporaceae of India. Dehra Dun: International Book Distributors, 1-309, 1996.

[12] K.M. Leelavathy, P.N. Ganesh. Polypores of Kerala. Delhi: Daya Publishing House, 1-165, 2000.

[13] Mycobank 2019. Fungal Databases. Nomenclature and species bank. [Accessed: 30/10/2019].

[14] G. Kaur, A.P. Singh, G.S. Dhingra. Diversity of genus Ganoderma in Punjab (India), Mycobiota, 7, 25-49, 2017.

[15] J.K. Brar, R. Kaur, G, Kaur, A.P. Singh, G.S. Dhingra. Taxonomic notes on the genus Ganoderma from Union Territory of Chandigarh, Kavaka, 51, 35-48, 2018.

[16] R. Kaur, H. Kaur, A.P. Singh, G.S. Dhingra. Some noteworthy additions to family Polyporaceae from Himachal Pradesh, Kavaka, 49, 10-14, 2017. 